\title{
A LOCAL MEAN VALUE THEOREM FOR THE COMPLEX PLANE
}

\author{
by J. M. ROBERTSON
}

(Received 18th November 1968)

\section{Introduction}

The equation

$$
f\left(z_{1}\right)-f\left(z_{0}\right)=f^{\prime}(z)\left(z_{1}-z_{0}\right)
$$

need not have a solution $z$ in the complex plane, even when $f$ is entire. For example, let $f(z)=e^{z}, z_{1}=z_{0}+2 k \pi i$. Thus the classical mean value theorem does not extend to the complex plane. McLeod has shown (2) that if $f$ is analytic on the segment joining $z_{1}$ and $z_{0}$, then there are points $w_{1}$ and $w_{2}$ on the segment such that $f\left(z_{1}\right)-f\left(z_{0}\right)=\left(z_{1}-z_{0}\right)\left(\lambda_{1} f^{\prime}\left(w_{1}\right)+\lambda_{2} f^{\prime}\left(w_{2}\right)\right)$ where $\lambda_{i} \geqq 0$ and $\lambda_{1}+\lambda_{2}=1$.

The purpose of this article is to give a local mean value theorem in the complex plane. We show that there is at least one point $z$ satisfying (1), which we will call a mean value point, near $z_{1}$ and $z_{0}$ but not necessarily on the segment joining them, provided $z_{1}$ and $z_{0}$ are sufficiently close. The proof uses Rouchés Theorem (1).

\section{The local mean value theorem}

Theorem. If $f(z)$ is analytic in a domain containing $z_{0}$, then there is a neighbourhood $N$ of $z_{0}$ such that if $z_{1}$ is in $N$ then (1) has at least one solution $z$ in $M=\left\{z:\left|z-z_{0}\right|<\left|z_{1}-z_{0}\right|\right\}$.

Proof. Write $f(z)=\sum_{k=0}^{\infty} \frac{f^{(k)}\left(z_{0}\right)}{k !}\left(z-z_{0}\right)^{k}$. Then assuming $z_{1} \neq z_{0}$, we may write by direct computation

$$
f^{\prime}(z)-\frac{f\left(z_{1}\right)-f\left(z_{0}\right)}{z_{1}-z_{0}}=\sum_{k=2}^{\infty} \frac{f^{(k)}\left(z_{0}\right)}{(k-1) !}\left\{\left(z-z_{0}\right)^{k-1}-\frac{1}{k}\left(z_{1}-z_{0}\right)^{k-1}\right\} .
$$

We now look for zeros of this expression and note that the zeros are independent of the constant and linear term of the power series. So, there is nothing to prove if $f$ is linear, since any $z$ is a zero of (2) in this case. Actually one can easily show that $z^{\prime}$ is a mean value point for $g$ and the pair $z_{1}, z_{0}$ if and only if $z^{\prime}$ is a mean value point for $g(z)+a+b z$ and the pair $z_{1}, z_{0}$. The proof is given in two cases since more can be said about the position of the mean value point if $f^{\prime \prime}\left(z_{0}\right) \neq 0$. 
Case 1: $f^{\prime \prime}\left(z_{0}\right) \neq 0$.

Set $g_{1}(z)=f^{\prime \prime}\left(z_{0}\right)\left\{\left(z-z_{0}\right)-\frac{1}{2}\left(z_{1}-z_{0}\right)\right\}=f^{\prime \prime}\left(z_{0}\right)\left\{z-\frac{1}{2}\left(z_{1}+z_{0}\right)\right\}$, the first term of the series in (2). Set

$$
g_{2}(z)=\sum_{k=3}^{\infty} \frac{f^{(k)}\left(z_{0}\right)}{(k-1) !}\left\{\left(z-z_{0}\right)^{k-1}-\frac{1}{k}\left(z_{1}-z_{0}\right)^{k-1}\right\} \text {. }
$$

Let $C=\left\{z:\left|z-\frac{1}{2}\left(z_{1}+z_{0}\right)\right|=\frac{1}{2}\left|z_{1}-z_{0}\right|\right\}$. Then, with $r=\frac{1}{2}\left|z_{1}-z_{0}\right|$, the following statements are valid for all $z$ on $C$ :

$\left|g_{1}(z)\right|=\left|f^{\prime \prime}\left(z_{0}\right)\right| \cdot r$,

$\left|g_{2}(z)\right| \leqq \sum_{k=3}^{\infty} \frac{\left|f^{k}\left(z_{0}\right)\right|}{(k-1) !}\left\{(2 r)^{k-1}+\frac{1}{k}(2 r)^{k-1}\right\}=\sum_{k=3}^{\infty} \frac{\left|f^{k}\left(z_{0}\right)\right|}{(k-1) !} \cdot \frac{k+1}{k} \cdot(2 r)^{k-1}$,

$\left|\frac{g_{2}(z)}{g_{1}(z)}\right| \leqq \frac{\sum_{k=3}^{\infty} \frac{\left|f^{k}\left(z_{0}\right)\right|}{(k-1) !} \frac{k+1}{k} \cdot(2 r)^{k-1}}{\left|f^{\prime \prime}\left(z_{0}\right)\right| \cdot r}$

$$
=\frac{1}{\left|f^{\prime \prime}\left(z_{0}\right)\right|} \sum_{k=3}^{\infty} \frac{\left|f^{(k)}\left(z_{0}\right)\right|}{(k-1) !} \frac{k+1}{k} \cdot 2^{k-1} r^{k-2}
$$

If the series $\sum_{k=0}^{\infty} \frac{f^{(k)}\left(z_{0}\right)}{k !}\left(z-z_{0}\right)^{k}$ converges for $\left|z-z_{0}\right|<R$, then the last series in (3) converges for $r<\frac{R}{2}$, and represents a continuous function of $r$ in that range with the value zero at $r=0$. Hence there is an $r_{0}>0$ such that if $r<r_{0}$ the series in (3) converges to a number less than one. So if $\left|z_{1}-z_{0}\right|<2 r_{0}$ we may say $\left|g_{2}(z)\right|<\left|g_{1}(z)\right|$ for all $z$ on $C$. Thus by Rouché's Theorem $g_{1}(z)$ and $g_{1}(z)+g_{2}(z)$ have the same number of zeros inside $C, g_{1}(z)$ has one zero at $\frac{z_{1}+z_{0}}{2}$. Hence there is one mean value point inside $C$.

Case 2: $0=f^{\prime \prime}\left(z_{0}\right)=\ldots=f^{(n-1)}\left(z_{0}\right)$, and $f^{(n)}\left(z_{0}\right) \neq 0$.

Set $g_{1}(z)=\frac{f^{(n)}\left(z_{0}\right)}{(n-1) !}\left(\left(z-z_{0}\right)^{n-1}-\frac{1}{n}\left(z_{1}-z_{0}\right)^{n-1}\right)$, the first non-vanishing term in (2). Set

$$
g_{2}(z)=\sum_{k=n+1}^{\infty} \frac{f^{(k)}\left(z_{0}\right)}{(k-1) !}\left(\left(z-z_{0}\right)^{k-1}-\frac{1}{k}\left(z_{1}-z_{0}\right)^{k-1}\right) .
$$

Let $C=\left\{z:\left|z-z_{0}\right|=\left|z_{1}-z_{0}\right|\right\}$ and $r=\left|z_{1}-z_{0}\right|$. Then for $z$ on $C$ we have

$$
\begin{aligned}
& \left|\frac{g_{2}(z)}{g_{1}(z)}\right| \leqq \frac{\sum_{k=n+1}^{\infty} \frac{\left|f^{(k)}\left(z_{0}\right)\right|}{(k-1) !}\left(r^{k-1}+\frac{1}{k} r^{k-1}\right)}{\frac{\left|f^{n}\left(z_{0}\right)\right|}{(n-1) !}\left(r^{n-1}-\frac{1}{n} r^{n-1}\right)} \\
& =\frac{n !}{(n-1)\left|f^{n}\left(z_{0}\right)\right|} \sum_{k=n+1}^{\infty} \frac{\left|f^{(k)}\left(z_{0}\right)\right|}{(k-1) !} \frac{k+1}{k} r^{k-n} .
\end{aligned}
$$


As in Case 1, we can argue this last series is convergent for $r<R$ and the series converges to zero at $r=0$. So there is an $r_{0}$ such that if $\left|z_{1}-z_{0}\right|<r_{0}$ then $\left|g_{2}(z)\right|<\left|g_{1}(z)\right|$ on $C$. Hence $g_{1}(z)$ and $g_{1}(z)+g_{2}(z)$ have the same number of zeros inside $C$. $g_{1}(z)=0$ has $n-1$ roots at $z_{0}+\left(z_{1}-z_{0}\right) \cdot n^{-1 /(n-1)}$ inside $C$. Hence there are $n-1$ mean value points (not necessarily distinct) inside $C$. This completes the proof.

\section{Example}

Let $f(z)=e^{z}$ so that Case 1 holds. In this case on $C$ we have $\left|g_{1}(z)\right|=\left|e^{z 0}\right| \cdot r$,

and

$\left|g_{2}(z)\right| \leqq\left|e^{z_{0}}\right| \sum_{k=3}^{\infty} \frac{k+1}{k} \frac{(2 r)^{k-1}}{(k-1) !}<\frac{4}{3}\left|e^{z_{0}}\right| \sum_{k=3}^{\infty} \frac{(2 r)^{k-1}}{(k-1) !}=\frac{4}{3}\left|e^{z_{0}}\right|\left(e^{2 r}-1-2 r\right)$.

Hence $\left|g_{1}(z)\right|>\left|g_{2}(z)\right|$ on $C$ provided $r>\frac{4}{3}\left(e^{2 r}-1-2 r\right)$ or $4 e^{2 r}-4-11 r<0$. This function has a minimum at $\frac{1}{2} \log 11 / 8$ which is greater than 0.15 . We know then that if $\left|z_{1}-z_{0}\right|<0.3$ then there is a mean value point for $e^{z}$ and the pair $z_{0}, z_{1}$ inside the circle with the segment $z_{0} z_{1}$ as a diameter.

\section{REFERENCES}

(1) E. HiLle, Analytic Function Theory, Vol. 1 (Ginn and Co., 1959).

(2) R. M. McLeod, Mean value theorems for vector valued functions, Proc. Edinburgh Math. Soc. (2) 14 (1965), 197-209.

WASHINGTON STate UNIVERSITY

PULLMAN, WASHINGTON-99163 